\section{Antineutrophil cytoplasmic antibodies: reporting and diagnostic strategies}

It was with great interest that we read the correspondence of Mahler and Fritzler ${ }^{1}$ on our recent European Vasculitis Study Group (EUVAS) study describing the performance of immunoassays for antineutrophil cytoplasmic antibodies (ANCA) in patients with ANCA-associated vasculitides (AAV). ${ }^{2}$ In their letter, Mahler and Fritzler raise some interesting points, mainly related to (i) test result interpretation and (ii) diagnostic strategies. Besides, they pointed out that in the EUVAS study only two indirect immunofluorescence (IIF) assays were included, a commercial assay from Inova Diagnostics and a 'home-made' assay. They suggested to perform studies with more than two IIF ANCA tests and especially to include assays that are most commonly used in diagnostic laboratories. In a concomitant publication, we presented data on two additional commercial IIF assays, one from Euroimmun and one from Medipan. ${ }^{3}$ Accordingly, we postulate that we included the most commonly used IIF assays. For example, IIF ANCA assays from Inova and Euroimmun are used by, respectively, $50 \%$ and $23 \%$ of the participants of the UK National External Quality Assessment Service (NEQAS) ANCA scheme (report September 2016). Our studies consistently showed that test characteristics of IIF were highly variable between assays. We envisaged that this variability was dependent on the substrates used and methods applied for ANCA IIF testing, that is, the use of only ethanol-fixed neutrophils versus the combination of ethanol-fixed and formalin-fixed neutrophils and HEp2 cells. ${ }^{3}$ Moreover, we also found that the overall performance of high-quality immunoassays was at least as good as the performance of IIF methods, even when applied on modern automated systems. These observations lead us to conclude that the current international guidelines on ANCA testing ${ }^{4}$ should be revised. A large Russian vasculitis centre has already abandoned IIF for ANCA testing several years ago ${ }^{5}$ and in Japan, immunoassays are used for the diagnosis of AAV without IIF in most cases (Y Arimura, personal communication).

As we foresee that proteinase-3 (PR3)-ANCA and myeloperoxidase (MPO)-ANCA will be increasingly used to screen for ANCA, it is important to fully understand the clinical value inherent in the test results generated by such assays. Usually, PR3-ANCA and MPO-ANCA are interpreted as positive or negative. However, a lot of information is lost when such dichotomous interpretation is used. In a local study, we previously showed that the likelihood for disease increases with increasing ANCA levels and that the use of likelihood ratios can improve the clinical usefulness. ${ }^{6}$ Using the large EUVAS dataset, we have performed in-depth studies on test result intervalspecific likelihood ratios for each of the assays included in the study. As we determined the test result intervals based on predefined specificities, we maximally harmonised test result interpretation between assays. These results have been submitted for publication (Bossuyt et al, submitted for publication). We highly appreciate the genuine interest of Mahler and Fritzler in this approach and hope that the information-when available-will be widely adopted by manufacturers and users of the assays.

Mahler and Fritzler also encouraged us to further expand the study by analysing the potential value of combining results from different tests, in particular IIF and immunoassays. Also this suggestion is relevant as it has previously been shown that combing tests may indeed increase the clinical utility. ${ }^{7}$ Here again, we have studied the value of combining different tests in detail using the EUVAS dataset and the results have been submitted for publication (Bossuyt et al submitted). Combining different tests can indeed increase the clinical utility, but the extent of the increase depends on the quality of the assay and the combination of assays. For example (based on data presented in ref. 2), in a simplified analysis using the single cut-off point proposed by the manufacturer, the area under the curve (AUC) of the Inova QuantaFlash PR3-ANCA and MPO-ANCA assay for AAV was 0.925 (95\% CI 0.909 to 0.940). The AUC of combining QuantaFlash with the best performing IIF ANCA assay included in the EUVAS study (an assay from Inova which combines ethanol and formalin fixation with antinuclear antibody (ANA) detection on HEp 2 cells) was not significantly different from the AUC of performing only QuantaFlash $(p=0.088)$. In contrast, the AUC of combining QuantaFlash with an immunoassay for MPO-ANCA and PR3-ANCA from Euroimmun on all samples was significantly different from the AUC of QuantaFlash alone $(p=0.01)$. The likelihood ratios for the different strategies (combinations) are given in table 1. This simplified approach, which does not take into account antibody levels, indicates the potential value of combining different tests. Moreover, these results suggest that combining two different immunoassays might be preferred to combining immunoassay with IIF. This can be even better appreciated by visual analysis of the results, as presented in figure 1 . In this figure, the individual test results for patients with AAV and controls are shown for the combination of QuantaFlash with IIF and for the combination of QuantaFlash with immunoassays from Euroimmun. It can be seen that controls that are single positive by either of two immunoassays generally have low antibody levels. By contrast, controls that are single positive by IIF might have high IIF antibody levels. This, together with the fact that there are three times more controls that are single positive by IIF than by the two immunoassays, argues for combining two highquality immunoassays rather than for combining immunoassay with IIF. Our data also show that combining different tests is mainly useful in case of low antibody levels by immunoassay (associated with a low likelihood ratio for disease) and much less useful for high antibody levels, as such results are associated with a high likelihood ratio for disease. This again illustrates the need for improved interpretation of test results that takes into account antibody levels.

Mahler and Fritzler ${ }^{1}$ suggested to combine immunoassays with IIF by referring to ANAs testing, in which combining IIF with immunoassays adds value. ${ }^{8}$ In ANA testing, IIF can pick up antibodies to relevant antigens that are not picked up by immunoassays. Such antibodies can be of high titre and are found in patients with systemic lupus erythematosus or systemic sclerosis. ${ }^{9}$ However, in AAV, PR3 and MPO are the main autoantigens and there is no need for IIF to detect antibodies to autoantigens other than MPO and PR3. Moreover, in patients with AAV, there is high concordance of antibody detection between immunoassays and between immunoassays and IIF. Seronegative patients are usually negative by immunoassays and by IIF. Pertinent to this, it should be pointed out that ANCA testing is only an adjunct for the diagnosis of AAV: clinicopathological features determine the diagnosis.

The EUVAS study focused on AAV and did not address ANCA testing for gastrointestinal diseases. As previously suggested by us $^{10}{ }^{11}$ and by Mahler and Fritzler, ${ }^{1}$ laboratories should differentiate between test requisitions for AAV versus other inflammatory conditions such as inflammatory bowel disease or autoimmune hepatitis. However, the clinical relevance of ANCA testing in non-AAV conditions is limited, as illustrated by the fact that ANCA test results are not incorporated in the respective diagnostic criteria. ${ }^{12-14}$ 
Table 1 Likelihood ratios (with 95\% Cls) for the cut-off point proposed by the manufacturer and for a combination of tests are given

\begin{tabular}{|c|c|c|c|c|}
\hline & $\mathrm{AAV}(\mathrm{n})$ & Control (n) & Likelihood ratio & $95 \% \mathrm{Cl}$ \\
\hline QuantaFlash (-) & 29 & 893 & 0.12 & 0.08 to 0.17 \\
\hline QuantaFlash (+) & 222 & 31 & 26 & 18 to 37 \\
\hline Euroimmun (-) & 27 & 894 & 0.11 & 0.08 to 0.16 \\
\hline Euroimmun $(+)$ & 224 & 30 & 27 & 19 to 39 \\
\hline QuantaFlash (-) IIF (-) & 23 & 854 & 0.10 & 0.07 to 0.15 \\
\hline QuantaFlash (-) IIF (+) & 6 & 39 & 0.57 & 0.24 to 1.32 \\
\hline QuantaFlash (+) IIF (-) & 5 & 13 & 1.42 & 0.51 to 3.93 \\
\hline QuantaFlash (+) IIF (+) & 217 & 18 & 44 & 28 to 70 \\
\hline QuantaFlash (-) Euroimmun (-) & 23 & 880 & 0.09 & 0.06 to 0.14 \\
\hline QuantaFlash (-) Euroimmun (+) & 6 & 13 & 1.70 & 0.65 to 4.42 \\
\hline QuantaFlash (+) Euroimmun (-) & 4 & 14 & 1.05 & 0.35 to 3.17 \\
\hline QuantaFlash (+) Euroimmun (+) & 218 & 17 & 47 & 29 to 75 \\
\hline Total & 251 & 924 & & \\
\hline
\end{tabular}

The number of patients and controls with a particular test result or combination of test result are given as well. The highest level of reactivity from the PR3-ANCA and MPO-ANCA determinations was selected for analysis. Data are from ref. 2.

The AUC of the Inova QuantaFlash PR3-ANCA and MPO-ANCA assay for AAV was 0.925 ( $95 \% \mathrm{Cl} 0.909$ to 0.940$)$. The AUC of combining QuantaFlash with an IIF ANCA assay was 0.94 ( $95 \%$ Cl 0.925 to 0.953$)$, which was not significantly different from the AUC of performing only QuantaFlash ( $p=0.088$ ) (method of Hanley and McNeil, MedCalc). The AUC of combining QuantaFlash with an immunoassay for MPO- and PR3-ANCA from Euroimmun on all samples was $0.943(95 \% \mathrm{Cl} 0.928$ to 0.955$)$, which was significantly different from the AUC of QuantaFlash alone $(p=0.01)$ (method of Hanley and McNeil, MedCalc).

AAV, ANCA-associated vasculitides; ANCA, antineutrophil cytoplasmic antibodies; MPO, myeloperoxidase.

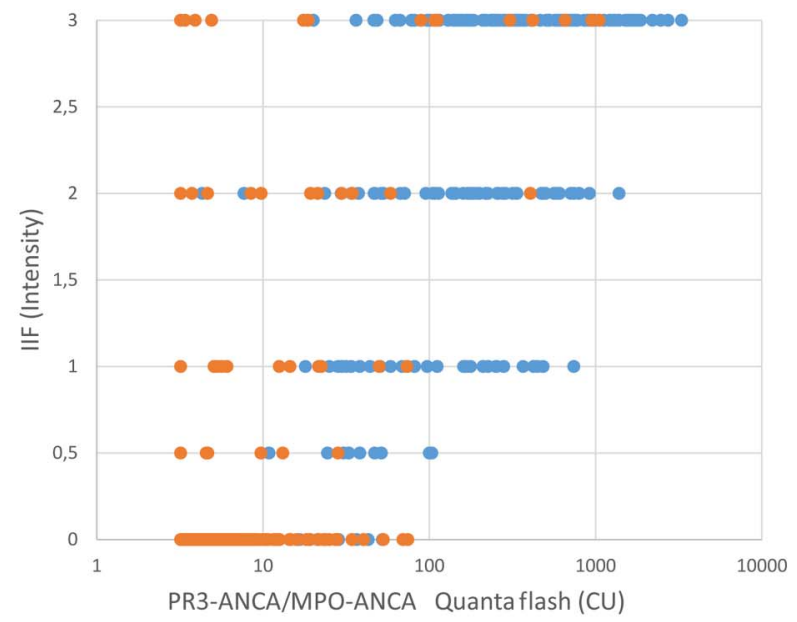

aAV econtro

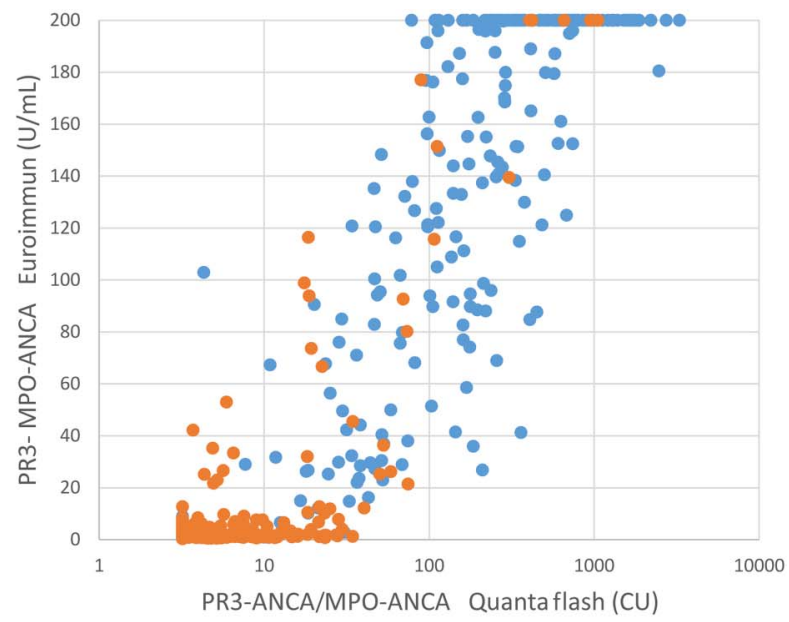

- AAV Control

Figure 1 Test results for antineutrophil cytoplasmic antibodies (ANCA) by QuantaFlash (Inova) and by ELISA (Euroimmun). The highest level of reactivity from the PR3-ANCA and myeloperoxidase (MPO)-ANCA determinations was selected for analysis. Cut-off point proposed by the manufacturer is $20 \mathrm{U} / \mathrm{mL}$ of $\mathrm{CU}$ for both assays. Data are from ref. 2.

Taken together, the data of the EUVAS study ${ }^{2}$ and additional data on test interpretation and testing strategies discussed above are a basis for a new international consensus on ANCA testing, which is currently in preparation. A strategy primarily based on antigen-specific assays seems to be supported by clinical practice in some laboratories, ${ }^{2}{ }^{5}$ but we consider it mandatory that such strategy is validated in a prospective study, potentially including a wider array of ANCA tests.

\section{Jan Damoiseaux, ${ }^{1}$ Elena Csernok, ${ }^{2}$ Niels Rasmussen, ${ }^{3}$} Jan-Willem Cohen Tervaert, ${ }^{4}$ Xavier Bossuyt ${ }^{5,6}$

${ }^{1}$ Central Diagnostic Laboratory, Maastricht University Medical Center, Maastricht, The Netherlands

${ }^{2}$ Department of Internal Medicine, Rheumatology and Immunology, University Teaching Hospital Kirchheim, Vasculitis-Center Tübingen-Kirchheim, Kirchheim-Teck, Germany

${ }^{3}$ Department of Autoimmunology \& Biomarkers, Statens Serum Institut, Kobenhavn, Denmark
${ }^{4}$ Maastricht University, Maastricht, The Netherlands

${ }^{5}$ Department of Microbiology and Immunology, KU Leuven, Leuven, Belgium

${ }^{6}$ Department of Laboratory Medicine, University Hospitals Leuven, Leuven, Belgium

Correspondence to Dr Xavier Bossuyt, Laboratory Medicine, University Hospitals Gasthuisberg, Herestraat 49, Leuven 3000, Belgium; Xavier.Bossuyt@uzleuven.be

JD and EC co-first authors.

Contributors XB and JD wrote the manuscript. NR, J-WCT and EC critically reviewed the manuscript and edited the manuscript.

Competing interests XB has been a consultant to Inova Diagnostics.

Ethics approval Ethics committee University Hospital Leuven, Belgium.

Provenance and peer review Commissioned; internally peer reviewed.

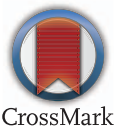


To cite Damoiseaux J, Csernok E, Rasmussen N, et al. Ann Rheum Dis 2017;76:e39.

Received 2 February 2017

Accepted 4 February 2017

Published Online First 8 March 2017

\section{SLinked}

http://dx.doi.org/10.1136/annrheumdis-2017-211157

Ann Rheum Dis 2017;76:e39. doi:10.1136/annrheumdis-2016-211171

\section{REFERENCES}

1 Mahler M, Fritzler. Commentary on the recent international multicentre study (EUVAS) on anti-neutrophil cytoplasmic antibodies. Ann Rheum Dis 2017:76:e36.

2 Damoiseaux J, Csernok E, Rasmussen N, et al. Detection of antineutrophil cytoplasmic antibodies (ANCAs): a multicentre European Vasculitis Study Group (EUVAS) evaluation of the value of indirect immunofluorescence (IIF) versus antigen-specific immunoassays. Ann Rheum Dis 2017;76:647-53.

3 Csernok E, Damoiseaux J, Rasmussen $\mathrm{N}$, et al. Evaluation of automated multi-parametric indirect immunofluorescence assays to detect antineutrophil cytoplasmic antibodies (ANCA) in granulomatosis with polyangiitis (GPA) and microscopic polyangiitis (MPA). Autoimmun Rev 2016:15:736-41.
4 Savige J, Gillis D, Benson E, et al. International consensus statement on testing and reporting of antineutrophil cytoplasmic antibodies (ANCA). Am I Clin Pathol 1999;111:507-13.

5 Novikov P, Smitienko I, Bulanov N, et al. Testing for antineutrophil cytoplasmic antibodies (ANCAs) in patients with systemic vasculitides and other diseases. Ann Rheum Dis 2017;76:e23.

6 Vermeersch P, Blockmans D, Bossuyt X. Use of likelihood ratios can improve the clinical usefulness of enzyme immunoassays for the diagnosis of small-vessel vasculitis. Clin Chem 2009;55:1886-8.

7 Vermeersch P, Vervaeke S, Blockmans D, et al. Determination of anti-neutrophil cytoplasmic antibodies in small vessel vasculitis: comparative analysis of different strategies. Clin Chim Acta 2008;397:77-81.

8 Bossuyt $X$, Fieuws $S$. Detection of antinuclear antibodies: added value of solid phase assay? Ann Rheum Dis 2014;73:e10

9 Op De Beeck K, Vermeersch P, Verschueren P, et al. Detection of antinuclear antibodies by indirect immunofluorescence and by solid phase assay. Autoimmun Rev 2011;10:801-8.

10 Avery TY, Bons J, van Paassen P, et al. Diagnostic ANCA algorithms in daily clinical practice: evidence, experience, and effectiveness. Lupus 2016;25:917-24.

11 Damoiseaux J, Csernok E, Rasmussen N, et al. Antineutrophil cytoplasmic antibodies: appropriate use and interpretation. Ann Rheum Dis 2017:76:e24.

12 Lohse AW. Diagnostic criteria for autoimmune hepatitis: scores and more. Dig Dis 2015:33(Suppl 2):47-52.

13 van Assche G, Dignass A, Panes J, et al. The second European evidence-based consensus on the diagnosis and management of Crohn's disease: definitions and diagnosis. J Crohns Colitis 2010;4:7-27.

14 Dignass A, Eliakim R, Magro F, et al. Second European evidence-based consensus on the diagnosis and management of ulcerative colitis part 1: definitions and diagnosis. J Crohns Colitis 2012:6:965-90. 